\title{
Satisfacción Laboral del Personal de Enfermería del Quirófano del Hospital Universitario José María Morales Meseguer. Murcia, España.
}

\author{
Job satisfaction of the nursing staff in University Hospital José María \\ Morales Meseguer. Murcia, Spain.
}

\author{
César Carrillo-García ${ }^{1 *}$, María Emilia Martínez-Roche², Catalina García-Maiquez ${ }^{3}$, \\ María Carmen Vivo-Molina ${ }^{4}$, Eduardo Melendreras-Montesinos ${ }^{5}$
}

\begin{abstract}
Resumen
La satisfacción del profesional sanitario es un elemento imprescindible en el proceso asistencial que se relaciona directamente con la calidad de los servicios sanitarios. Tanto la satisfacción como la motivación laboral son importantes elementos de estudio debido a las consecuencias que tienen para los trabajadores y su repercusión en el funcionamiento de las diferentes organizaciones y en la calidad de los servicios prestados. Objetivo: Evaluar la satisfacción laboral del personal de enfermería del área quirúrgica de un Hospital Universitario de segundo nivel. Material y método: Estudio observacional, analítico y transversal realizado al personal de enfermería del área quirúrgica. La recolección de datos se realizó en el primer trimestre del año 2015. Se utilizó como instrumento la NTP 394: Satisfacción Laboral: escala general de satisfacción. Análisis de datos: pruebas no paramétricas para 2 muestras o k muestras según la comparación. Resultados: Los resultados muestran una tasa de participación del $89,65 \%$. Respecto a la satisfacción general, la media de los participantes se sitúa en $58,76(D T=16,64)$, respecto a la satisfacción extrínseca e intrínseca la media es de $31,42(D T=9,04)$ y $27,34(D T=8,22)$ respectivamente. "La estabilidad en el empleo" es el factor en el que los profesionales están más satisfechos, mientras que "el modo en que se está gestionando" es el factor con el que se encuentran menos satisfechos. Los principales hallazgos reflejan niveles medios de satisfacción entre los profesionales del quirófano del Hospital Universitario, siendo necesario desde el área de gestión, intervenir en aquellos factores en los que los profesionales se muestran más insatisfechos.
\end{abstract}

Palabras clave: Satisfacción laboral, hospitales universitarios, enfermería de quirófano.

\section{Abstract}

Satisfaction of the sanitary professionals is a very important issue in the care process, and it is directly related to the quality of sanitary services. Satisfaction and workers motivation are two important elements

1 Doctor en Enfermería. Coordinación de Desarrollo Profesional. Dirección General de Recursos Humanos del Servicio Murciano de Salud. Consejería de Salud de la Región de Murcia. Profesor Asociado Facultad de Enfermería de la Universidad de Murcia. Murcia. España.

2 Doctora en Pedagogía. Departamento de Enfermería. Facultad de Enfermería de la Universidad de Murcia. Murcia. España.

3 Enfermera. Hospital Universitario José María Morales Meseguer. Murcia. España.

4 Licenciada en Ciencias y Técnicas Estadísticas. Hospital Universitario José María Morales Meseguer. Murcia. España.

5 Facultativo Especialista de Área. Cirugía Ortopédica y Traumatología. Hospital Universitario José María Morales Meseguer. Murcia. España.

Correspondencia: César Carrillo García. Correo electrónico: cesar.carrillo@carm.es

Recibido: 10/12/2015 - Revisado: 18/05/16 - Aceptado: 30/08/2016 
to study. Consequences of these elements over the workers and their repercussion in the performance of the different organizations and in the quality of the services offered must be studied. Objective: to evaluate the satisfaction of the nursing staff in the surgery area in a second level University Hospital. Material and method: Observational, analytic and transversal study to the nursing staft in the surgical area. The data collection was done in the first quarter of 2015. It was used as instrument the NTP 394: job satisfaction: general scale of satisfaction. Analysis of data: nonparametric tests for two samples o $k$ samples according to comparison. Results: The results show a participation rate of $89,65 \%$. The average among participants was 58,76 (DT: 16,64) with regard to general satisfaction. Regard extrinsic and intrinsic satisfaction de average was 31,42 (DT: 9,04) and 27,34 (DT: 8,22) respectively. "Job stability" was the factor in which the professionals were most satisfied; "the way things are managed" won the lowest satisfaction. The main findings reflect a medium level of satisfaction among the sanitary workers in the surgical area in University Hospital. It's necessary from the management area intervene in those factors that professional are less satisfied.

Palabras clave: job satisfaction, hospital university, operating room nursing.

\section{Introducción}

Se define el trabajo como un conjunto de actividades humanas, con carácter productivo y retribuido con las que se obtiene o presta productos o servicios. Para realizar dicha actividad, la persona aporta habilidades y conocimientos obteniéndose a su vez una recompensa material, psicológica y/o social ${ }^{(1)}$.

Si nos centramos en la satisfacción laboral, se ha intentado explicar desde numerosas teorías o enfoques, sobre todo desde el campo de la motivación dado el papel central que la satisfacción juega en muchas de ellas, básicamente se centran en el concepto de discrepancia o ajuste ya que cuanto mayor sea la distancia entre lo que se quiere conseguir y lo que se obtiene del trabajo, menor será la satisfacción laboral ${ }^{(2)}$.

Podemos aproximarnos a la definición de satisfacción laboral como un conjunto de emociones y actitudes que la persona experimenta hacia su trabajo. La satisfacción laboral engloba tanto actitudes como emociones, considerándola como una actitud compuesta por elementos afectivos, cognitivos y conductuales que pueden variar en consistencia y magnitud, pueden ser obtenidos de diferentes fuentes y cumplen distintas funciones para el individuo ${ }^{(2)}$.

La justificación de este estudio viene avalada por la importancia que la satisfacción laboral tiene en los servicios de salud como un indicador de calidad tanto de los servicios prestados como de la organización interna de las instituciones sanitarias ${ }^{(3)}$ y como un indicador de la calidad de vida laboral ${ }^{(4)}$, asimismo, se ha señalado que las personas que se encuentran motivadas y satisfechas en el desarrollo de su actividad laboral, aumentan su rendimiento y la calidad de los servicios que prestan ${ }^{(5)}$, por lo que la satisfacción laboral también es un factor determinante en la satisfacción de los usuarios ${ }^{(6)}$. La importancia de medir la satisfacción laboral en cualquier contexto incluido el sanitario, ha sido señalada por numerosos autores ${ }^{(3-6)}$.

El objetivo general del estudio fue evaluar la satisfacción de la vida laboral del personal sanitario (enfermeros y auxiliares de enfermería) del área quirúrgica de un Hospital Universitario. Asimismo, se plantearon como objetivos específicos de- terminar el nivel de satisfacción en función de las variables socio-demográficas y de identificación; analizar los niveles de satisfacción en función de los factores intrínsecos y extrínsecos y comparar el grado de satisfacción entre las distintas categorías laborales.

\section{Material y método}

El diseño de la investigación se define como la planificación de la estrategia para conseguir los objetivos de la investigación $^{(7)}$. Para la realización de este estudio se planteó el diseño desde un enfoque cuantitativo, descriptivo, observacional, de diseño no experimental y de tipo corte transversal, valorando las características de un grupo de profesionales sanitarios realizando una única medición a la población de estudio en una fecha concreta. La población de estudio la constituyen todos los profesionales de enfermería y auxiliares de enfermería del área quirúrgica del Hospital Universitario José María Morales Meseguer $(\mathrm{N}=60)$. Los criterios de inclusión fueron todos los enfermeros y auxiliares del área quirúrgica del Hospital y como criterios de exclusión el personal de baja en el momento de cursar la encuesta, y profesionales que voluntariamente decidieron no participar; una vez aplicados los criterios de inclusión y exclusión la población de estudio quedó constituida por 58 profesionales.

La medición de las variables realizó utilizando los datos primarios recogidos de forma directa por el equipo investigador mediante cuestionarios autoadministrados entregados al personal a estudio. Las variables sociolaborales y de identificación cuantitativas que se consideraron fueron la edad y antigüedad, y, las cualitativas fueron sexo (hombre o mujer), vinculación (propietario, interino, comisión de servicio o acumulación de tareas), servicio (quirófano programado o de urgencias) y categoría profesional (enfermero/a o auxiliar de enfermería).

Como instrumento de recogida de datos se utilizó la escala NTP 394 (satisfacción laboral: escala general de satisfacción). Este cuestionario incluye dos dimensiones o subescalas, por un lado una subescala de factores intrínsecos compuesta por 7 ítems y que aborda aspectos como el reconocimiento obtenido por el trabajo, responsabilidad, promoción, etc., y 
otra subescala de factores extrínsecos que la componen 8 ítems y que aborda aspectos relativos a la organización del trabajo como el horario, la remuneración, las condiciones físicas del trabajo, etc. En total la escala se compone de 15 ítems, siendo valorado cada uno mediante una escala Likert de 7 puntos de anclaje. Esta es una escala aditiva en la cual la puntuación total se obtiene de la suma de los posicionamientos del encuestador en cada uno de los 15 ítems, asignando un valor de 1 (muy insatisfecho) y correlativamente hasta asignar un valor de 7 (muy satisfecho). La puntuación total de la escala oscila entre 15 y 105, de manera que una mayor puntuación refleja una mayor satisfacción general, la corrección de las subescalas es idéntica a la de la escala general si bien, debido a su menor longitud, sus valores oscilan entre 7-49 para la satisfacción intrínseca y 8-56 para la satisfacción extrínseca. Respecto al nivel de fiabilidad de la escala medido con el Alpha de Cronbach es de entre 0,85 y 0,88 para la satisfacción general, entre 0,79 y 0,85 para la intrínseca y entre 0,74 y 0,78 para la extrínseca ${ }^{(8)}$.

La presente investigación se realizó bajo el cumplimiento de la Ley Orgánica 15/1999, de 13 de diciembre, de Protección de Datos de carácter Personal para cumplir con los requisitos legales del estudio, asimismo, se pidió autorización por escrito al Sr. Director Gerente del Hospital Morales Meseguer para la realización del presente estudio y a los autores de la escala NTP 394: satisfacción laboral (escala general de satisfacción), publicada por el Instituto Nacional de Seguridad e Higiene en el trabajo, adscrito al Ministerio de Trabajo e Inmigración de España.

Tras los permisos, se presentó el proyecto de trabajo en una sesión de enfermería para solicitar la participación del personal en la misma. La encuesta se entregó en el primer trimestre de 2015 tanto en el quirófano programado como en el de urgencias, junto al cuestionario se entregó una hoja explicativa y un sobre. El cuestionario debía ser diligenciado y depositado en el sobre e inmediatamente proceder a sellar para garantizar el anonimato de la misma de los encuestados. La encuesta fue recogida en un plazo de cuatro semanas tras su entrega por el equipo investigador.

El análisis de resultados se realizó con el paquete informático SPSS (v.20.0), y junto al análisis descriptivo de la escala NTP 394 se realizó un análisis adicional estadístico de asociación de variables a fin de determinar si existen o no relaciones de dependencia entre los diferentes ítems de la escala y las variables sociodemográficas y laborales contempladas en el estudio. Para dicho análisis se utilizaron las técnicas no paramétricas U de Mann-Whitney y Kruskall-Wallis según fueran las comparaciones para dos o $\mathrm{k}$ muestras independientes. Las comparaciones se realizaron por sexo, edad, categoría profesional, antigüedad y vinculación con el servicio del área quirúrgica.

\section{Resultados}

Se obtuvo una tasa de participación del 89,65\%. La fiabilidad obtenida para esta muestra, medida con el Alpha de Cronbach para la satisfacción general fue de $\alpha=0.88$, mientras que para la satisfacción intrínseca y extrínseca fue de $\alpha=0.81$ y $\alpha=0.75$ respectivamente.

\section{Variables sociolaborales}

Los datos de las variables sociolaborales estudiadas se exponen en la tabla 1. Estando el perfil sociolaboral mayoritario conformado por una mujer de mediana edad, con plaza en propiedad, adscrita al quirófano programado, una edad media de 45,88 años y una experiencia profesional media de 12,15 años.

Tabla 1. Características sociolaborales de la muestra.

\begin{tabular}{lcc}
\hline & Porcentaje & (n) \\
\hline Sexo & & \\
Hombre & $71,2 \%$ & 37 \\
Mujer & $26,9 \%$ & 14 \\
Perdidos & $1,9 \%$ & 1 \\
Vinculación & & \\
Propietario & $76,9 \%$ & 26 \\
Interino & $7,7 \%$ & 10 \\
Comisión de Servicio & $9,6 \%$ & 7 \\
Acumulación de Tareas & $3,8 \%$ & 8 \\
Perdidos & $1,9 \%$ & 1 \\
Servicio & & \\
Quirófano Programado & $58,8 \%$ & 30 \\
Quirófano de Urgencias & $54,2 \%$ & 22 \\
Categoría Profesional & & \\
Enfermero & $76,9 \%$ & 40 \\
Auxiliar de Enfermería & $19,2 \%$ & 10 \\
Perdidos & $3,8 \%$ & 2 \\
\hline
\end{tabular}

Respecto a la edad media de los profesionales es de 45,88 años $(\mathrm{DT}=7,25)$, en relación a la antigüedad media en el Servicio de Quirófano es de 12,15 (DT=6,40), lo que indica diferencias importantes entre los trabajadores consultados en cuanto al número de años que llevan trabajando en el quirófano. Asimismo, la edad media de los profesionales en función del sexo muestran que la edad media de los hombres es de 46,71 (DT=6,41) y en las mujeres muy similar, 45,54 (DT=7,62). En relación a la antigüedad en el Servicio de Quirófano, la de los hombres es de 15,07 (DT=5,47), mientras que la de las mujeres es de 10,91 (DT=6,43).

\section{Satisfacción Laboral}

En primer lugar analizamos el nivel medio de satisfacción laboral y con el apoyo de la estructura original de la Escala General de Satisfacción se abordó un nivel de análisis diferenciando entre satisfacción intrínseca, extrínseca y satisfac- 
R.F.S Revista Facultad de Salud

Julio - Diciembre de 2016;8(2):9-15 ción general. Respecto a la satisfacción general, la medía de los participantes se sitúa en $58,76(\mathrm{DT}=16,64)$, por su parte, la satisfacción intrínseca es del 27,34 (DT=8,22), en tanto que la obtenida en la satisfacción extrínseca se sitúa en 31,42 (DT=9,04).

Realizando un análisis pormenorizado de los quince ítems que componen el cuestionario en la tabla 2 se describen para cada ítem la media y desviación típica.

\section{Análisis de la Satisfacción Laboral}

Por último, se incluyó un análisis adicional con el fin de determinar si se observaban diferencias significativas entre los distintos ítems del cuestionario y las variables sociodemográficas estudiadas.

En las variables sociodemográficas se encontraron diferencias significativas en la antigüedad, siendo mayor en los hombres que en la mujeres (15.07 vs 10.91), con un valor de $\mathrm{p}=0.04$. Asimismo, también se hallaron diferencias significativas en la antigüedad por categoría laboral, siendo mayor en los enfermeros/as que en auxiliares de enfermería (13.36 vs 8.8), con un valor de $p=0.041$. No se encontraron diferencias significativas en el resto de variables sociodemográficas.

Atendiendo al sexo de los profesionales encuestados, se encontraron diferencias significativas en "tu salario" y "el modo en que se está gestionando". En la tabla 3 se muestran las diferencias entre la satisfacción laboral y sexo.

Según la edad de los profesionales, se encontraron diferencias significativas en "la variedad de las tareas que realizas en tu trabajo". En la tabla 4 se muestran las diferencias entre la satisfacción laboral y edad.

Respecto a la categoría laboral se encontraron diferencias significativas en "la libertad para elegir tu propio método de trabajo". En la tabla 5 se muestran las diferencias entre la satisfacción laboral y categoría laboral.

Tabla 2. Medias y desviaciones típicas (DT) de las características sociolaborales de la muestra para cada ítems.

\begin{tabular}{lcc}
\hline Ítems & Media & Desviación típica \\
\hline 1. Condiciones físicas de trabajo & 3,84 & 1,53 \\
2. Libertad para elegir tu propio método de trabajo & 3,92 & 1,78 \\
3. Tus compañeros de trabajo & 5,40 & 1,56 \\
4. Reconocimiento que obtienes por el trabajo bien hecho & 3,59 & 1,56 \\
5. Tu superior inmediato & 4,41 & 2,06 \\
6. Responsabilidad que se te ha asignado & 4,84 & 1,17 \\
7. Tu salario & 3,56 & 1,63 \\
8. Las posibilidades de utilizar tus capacidades & 4,14 & 1,62 \\
9. Relación entre dirección y trabajadores & 2,63 & 1,41 \\
10. Tus posibilidades de promocionar & 3,43 & 1,45 \\
11. El modo en el que se está gestionando & 2,61 & 1,53 \\
12. La atención que se presta a las sugerencias que haces & 3,31 & 1,60 \\
13. Tu horario de trabajo & 4,51 & 1,81 \\
14. La variedad de tareas que realizas en tu trabajo & 5,14 & 1,28 \\
15. Tu estabilidad en el empleo & 5,41 & 1,71
\end{tabular}

Tabla 3. Diferencias entre la satisfacción laboral y sexo.

\begin{tabular}{lcccc}
\hline Ítems & Sexo & $\mathrm{N}$ & Rango promedio & p-Valor \\
\hline Tu salario & Hombre & 14 & 33,54 & 0,014 \\
& Mujer & 36 & 22,38 & \\
& Hombre & 14 & 37,32 & 0,001 \\
El modo en que se está gestionando & Mujer & 37 & 21,72 & \\
\hline
\end{tabular}


Tabla 4. Diferencias entre la satisfacción laboral por grupo de edad.

\begin{tabular}{lcccc}
\hline Ítems & Grupo de edad & N & Rango promedio & p-Valor \\
\hline La variedad de las & $<41$ & 15 & 30,00 & \\
tareas que realizas en & $41-50$ & 20 & 27,23 & 0,016 \\
tu trabajo & $51-60$ & 14 & 16,46 & \\
\hline
\end{tabular}

Tabla 5. Diferencias entre la satisfacción laboral por categoría laboral.

\begin{tabular}{lcccc}
\hline Ítems & Categoría laboral & $\mathbf{N}$ & Rango promedio & p-Valor \\
\hline $\begin{array}{l}\text { La libertad para elegir } \\
\text { tu propio método } \\
\text { de trabajo }\end{array}$ & Enfermero/a & 40 & 22,71 & \\
\hline
\end{tabular}

Por último y respecto a servicio (quirófano programado/ quirófano de urgencias), se encontraron diferencias significativas en "condiciones físicas del trabajo", "libertad para elegir tu propio método de trabajo", "tus compañeros de trabajo", "reconocimiento que obtienes por el trabajo bien hecho", "tu superior inmediato", "tu salario", "las posibilidades de utilizar tus capacidades", "relación entre dirección y trabajadores", "modo en el que se está gestionando", y "la atención que se presta a las sugerencias que haces". En la tabla 6 se muestran las diferencias entre la satisfacción laboral y servicio.

\section{Discusión}

El nivel de participación obtenido es superior al de otros estudios $^{(9,10)}$. Si se hace énfasis en el análisis descriptivo de la muestra, y comenzando por la variable sexo, se puede afirmar que se mantiene la tendencia de la intensa feminización de las profesiones sanitarias tal y como manifiestan otros autores ${ }^{(10-12)}$. Para el resto de variables, el perfil sociodemográfico estaría representado por profesionales de edad media, y propietarios en relación a la vinculación, tal y como sucede en otros estudios ${ }^{(9-13)}$.

En relación al objetivo general planteado, los resultados de satisfacción general, intrínseca y extrínseca son inferiores a los obtenidos en 2010 por profesionales de todos los servicios del mismo hospital y utilizando igualmente la escala NTP 394: satisfacción laboral ${ }^{(11)}$. El porqué de ésta diferencia sería motivo de estudio, aunque se llega a pensar que el incremento de la jornada laboral, crisis económica, etc. pueden justificar esta disminución en los niveles de satisfacción, por lo que una investigación más a fondo sobre las características del puesto de trabajo en los profesionales de enfermería del área quirúrgica permitiría identificar los factores generadores de satisfacción e insatisfacción a fin de poner en marcha desde la dirección medidas que mejorasen la satisfacción.
Los profesionales muestran una mayor satisfacción con el elemento "tu estabilidad en el empleo" y "tus compañeros de trabajo", estando vinculado el primer aspecto al elevado número de profesionales con plaza en propiedad; y en relación al segundo aspecto, queda de manifiesto la importancia de las relaciones personales en el ámbito laboral, no solo como fuente de satisfacción, ya que también las relaciones interprofesionales son consideradas como protectoras frente a los efectos del estrés y desgaste profesional. Este aspecto se manifiesta igualmente en profesionales de distintos hospitales e incluso en servicios de emergencias ${ }^{(14,15)}$. En el extremo opuesto se encuentran los cuatro ítems que obtienen los niveles de satisfacción más bajos como "tus posibilidades de promocionar", "la atención que se presta a las sugerencias que haces", "relación entre dirección y trabajadores" y "el modo en el que se está gestionando", aspectos que están en consonancia con otros estudios que abarcan todos los servicios hospitalarios e incluso en unidades de emergencias y utilizando tanto el mismo instrumento de medida como otro distinto ${ }^{(11-13)}$. Dado que en la actual estructura organizativa de los servicios de salud las posibilidades de promoción se obtienen por antigüedad, no estando valorados los méritos profesionales; y respecto a la gestión, al ser un considerado por el trabajador como un aspecto politizado, hace que se mantenga como un elemento negativo tal y como comentan otros autores ${ }^{(11)}$.

Como implicaciones prácticas del presente estudio se considera esencial establecer acciones que favorezcan y faciliten la comunicación entre los profesionales y la dirección, con la finalidad de identificar las áreas de insatisfacción y satisfacción, a fin de planificar y realizar los cambios organizativos necesarios ${ }^{(16)}$, asimismo, el diseño de acciones de mejora por parte de los gestores para corregir los aspectos que producen mayor insatisfacción en los profesionales debe ser una actividad habitual y prioritaria ${ }^{(17)}$.

En cuanto a las limitaciones de este estudio es preciso matizar, en primer lugar, que se tuvo que disponer de una muestra 
Tabla 6. Diferencias entre la satisfacción laboral por tipo de servicio.

\begin{tabular}{|c|c|c|c|c|}
\hline Ítems & Servicio & $\mathbf{N}$ & Rango promedio & p-Valor \\
\hline \multirow[t]{2}{*}{ Condiciones físicas de trabajo } & Qx Prog* & 28 & 21,36 & \multirow[t]{2}{*}{0,035} \\
\hline & Qx Urg* & 21 & 29,86 & \\
\hline \multirow[t]{2}{*}{ Libertad para elegir tu propio método de trabajo } & Qx Prog & 29 & 20,14 & \multirow[t]{2}{*}{0,002} \\
\hline & Qx Urg & 21 & 32,9 & \\
\hline \multirow[t]{2}{*}{ Tus compañeros de trabajo } & Qx Prog & 29 & 21,24 & \multirow[t]{2}{*}{0,012} \\
\hline & Qx Urg & 21 & 31,38 & \\
\hline \multirow[t]{2}{*}{ Reconocimiento que obtienes por el trabajo bien hecho } & Qx Prog & 30 & 20,97 & \multirow[t]{2}{*}{0,003} \\
\hline & Qx Urg & 21 & 33,19 & \\
\hline \multirow[t]{2}{*}{ Tu superior inmediato } & Qx Prog & 30 & 20,4 & \multirow[t]{2}{*}{0,001} \\
\hline & Qx Urg & 21 & 34 & \\
\hline \multirow[t]{2}{*}{ Tu salario } & Qx Prog & 29 & 20,83 & \multirow[t]{2}{*}{0,007} \\
\hline & Qx Urg & 21 & 31,95 & \\
\hline \multirow[t]{2}{*}{ Las posibilidades de utilizar tus capacidades } & Qx Prog & 29 & 21,55 & \multirow{2}{*}{0,022} \\
\hline & Qx Urg & 21 & 30,95 & \\
\hline \multirow[t]{2}{*}{ Relación entre dirección y trabajadores } & Qx Prog & 30 & 19,62 & \multirow[t]{2}{*}{0} \\
\hline & Qx Urg & 21 & 35,12 & \\
\hline \multirow[t]{2}{*}{ El modo en el que se está gestionando } & Qx Prog & 30 & 19,32 & \multirow[t]{2}{*}{0} \\
\hline & Qx Urg & 21 & 35,55 & \\
\hline \multirow[t]{2}{*}{ La atención que se presta a las sugerencias que haces } & Qx Prog & 30 & 21,42 & \multirow[t]{2}{*}{0,007} \\
\hline & Qx Urg & 21 & 32,55 & \\
\hline
\end{tabular}

* Nota: Qx Prog: Quirófano Programado, Qx Urg: Quirófano Urgencias.

de profesionales seleccionada de forma no aleatoria. Al ser recogidos los datos mediante cuestionario quedó a criterio de los participantes la sinceridad de las respuestas. Debido a que la población total de profesionales del quirófano no es elevada, un mayor tamaño de la muestra procedente de otros hospitales pude haber permitido la comparación de los resultados obtenidos. No obstante, estas insuficiencias no invalidan los resultados obtenidos para ser comparados con los obtenidos en estudios previos.

\section{Conclusiones}

Los resultados de este estudio evidencian que los profesionales de enfermería y auxiliares de enfermería, que trabajan en los quirófanos programados y de urgencias, presentan niveles de satisfacción medios, siendo estos a su vez, inferiores a estudios similares realizados cinco años antes en otras unidades del mismo hospital.

Los profesionales dejan de manifiesto que tanto la estabilidad en el empleo como los compañeros de trabajo son los aspectos que mayor satisfacción generan en estos profe- sionales, quedando patente la importancia de las relaciones interpersonales y el potencial humano como pilar básico en el ejercicio de la actividad laboral de los profesionales sanitarios. En sentido inverso, los aspectos profesionales que presentan mayor insatisfacción es la relación de los trabajadores con la dirección, así como el modo de gestión.

Dados los resultados de este estudio, sería conveniente que desde la Dirección de Enfermería se estableciera un plan de comunicación con los profesionales a fin de establecer estrategias que permitan elevar los niveles de satisfacción laboral de los profesionales, lo que conllevaría un aumento de la calidad asistencial.

\section{Bibliografía}

1. Peiró JM, Prieto F. Tratado de Psicología del Trabajo. Madrid: Síntesis; 1996.

2. Sáez MC. Satisfacción Laboral. En: García M, ed. Psicología del Trabajo y de las Organizaciones. Murcia: Diego Marín; 1999. P.131-146. 
3. European Foundation for Quality Management. Modelo Europeo de Excelencia. Madrid: Club Gestión de la Calidad; 1999.

4. Peiró JM, Silla I, Sanz T, Rodríguez J, García J. Satisfacción laboral de los profesionales de Atención Primaria. Psiquis: Revista de Psiquiatría, Psicología Médica y Psicosomática. 2004; 25: 5-16.

5. Robles M, Dierssen T, Martínez E, Herrera P, Díaz AR, Llorca J. Variables relacionadas con la satisfacción laboral: un estudio transversal a partir de modelo EFQM. Gaceta Sanitaria. 2005; 19: 127-134.

6. García J, Ranchal J, Biedma L, Serrano del Rosal, R. Preferencias en expectativas de los médicos residentes en Andalucía. Córdoba: Instituto de Estudios Sociales Avanzados; 2008.

7. Cea MA. Metodología cuantitativa. Estrategias y técnicas de investigación social. Madrid: Síntesis; 2001.

8. Instituto Nacional de Seguridad e Higiene en el Trabajo (ES). NTP:394: Satisfacción Laboral: escala general de satisfacción. 1995.

9. Carrillo-García C, Solano-Ruiz MC, Martínez Roche ME, Gómez-García $\mathrm{Cl}$. Job satisfacción among health care workers: The role of gender and age. Revista LatinoAmericana de Enfermagen. 2013; 21 : 1314-1320. Doi: 10.1590/0104-1 169.3224.2369.

10. García A, Moro MN, Medina M. Evaluación y dimensiones que definen el clima y la satisfacción laboral en el personal de Enfermería. Revista de Calidad Asistencia. 2010; 25: 207-214. Doi: 10.1016/i.cali.2010. 02.003.

11. Carrillo-García C, Martínez-Roche ME, Gómez-García $\mathrm{Cl}$, Meseguer-de-Pedro M. Satisfacción laboral de los profesionales sanitarios de un hospital universitario: análisis general y por categorías. Anales de Psicología. 2015; 31: 645-650. Doi: 10.6018/analesps.31.2. 169791.

12. Alves PC, Neves VF, Dela Coleta MF, Oliveira AF. Evaluation of well-being at work among nursing professionals at a University Hospital. Revista LatinoAmericana de Enfermagem. 2012; 20: 701-709.

13. Carrillo-García C; Martínez-Roche ME, Vivo-Molina MC, Quiñonero-Méndez F, Gómez-Sánchez R, Celdrán-Gil F. Satisfacción laboral de los médicos de las unidades móviles de emergencia y del centro coordinador de la gerencia de urgencias y emergencias 061. Región de Murcia. Revista de Calidad Asistencial. 2014; 29: 341 349. doi: 10.1016/i.cali.2014.11.006.

14. Sánchez A, De Lucas N, García Ochoa M, Sánchez C, Jiménez JA, Bustinza $A$. Estrés laboral en los profesionales de un servicio de emergencias prehospitalario. Emergencias. 2001; 13:170-175.

15. Topa G, Moriano JA, Morales JF. Acoso laboral entre profesionales de enfermería: El papel protector de la identidad social en el trabajo. Anales de Psicología. 2009; 25: 266-276

16. Miret C, Martínez-Larrea A. El profesional en urgencias y emergencias: Agresividad y burnout. Anales del Sistema Sanitario de Navarra. 2010; 33: 193-201.

17. Ríos-Rísquez MI, Godoy Fernández C, Sánchez-Meca J. Síndrome de quemarse por el trabajo, personalidad resistente y malestar psicológico en personal de enfermería. Anales de Psicología. 2011; 27: 71-79. 